\title{
The Potential of the Combination of CRISPR/Cas9 and Pluripotent Stem Cells to Provide Human Organs from Chimaeric Pigs
}

\author{
Wanyou Feng ${ }^{1}$, Yifan Dai ${ }^{2}$, Lisha Mou ${ }^{1, *}$, David K. C. Cooper ${ }^{3}$, Deshun Shi ${ }^{4}$ \\ and Zhiming Cai ${ }^{1, *}$
}

1 Shenzhen Key Laboratory of Xenotransplantaton, State and Local Joint Cancer Genome Clinical Application of Key Technology Laboratory, Shenzhen Second People's Hospital, First Affiliated Hospital of Shenzhen University, Shenzhen 518039, China;

E-Mail: fwanyou@gmail.com

2 Jiangsu Key Laboratory of Xenotransplantation, Nanjing Medical University, Nanjing 210029, China; E-Mail: daiyifan@njmu.edu.cn

3 Thomas E. Starzl Transplantation Institute, University of Pittsburgh Medical Center, Pittsburgh, PA 15261, USA; E-Mail: coopdk@upmc.edu

4 State Key Laboratory of Conservation \& Utilization of Subtropical Agro-Bioresources, Guangxi University, Nanning 530005, China; E-Mail: ardsshi@gxu.edu.cn

* Authors to whom correspondence should be addressed;

E-Mails: lishamou@gmail.com (L.M.); caizhiming2000@163.com (Z.C.);

Tel./Fax: +86-755-8355-7380 (L.M. \& Z.C.).

Academic Editor: Izuho Hatada

Received: 28 January 2015 / Accepted: 16 March 2015 / Published: 23 March 2015

\begin{abstract}
Clinical organ allotransplantation is limited by the availability of deceased human donors. However, the transplantation of human organs produced in other species would provide an unlimited number of organs. The pig has been identified as the most suitable source of organs for humans as organs of any size would be available. Genome editing by RNA-guided endonucleases, also known as clustered regularly interspaced short palindromic repeat (CRISPR/Cas9), in combination with induced pluripotent stem cells (iPSC), may have the potential to enable the creation of human organs from genetically-modified chimaeric pigs. These could potentially provide an unlimited supply of organs that would not be rejected by the recipient's immune system. However, substantial research is needed to prove that this approach will work. Genetic
\end{abstract}


modification of chimaeric pigs could also provide useful models for developing therapies for various human diseases, especially in relation to drug development.

Keywords: CRISPR/Cas9; genome editing; iPSC; pigs; chimaeric; xenotransplantation

\section{Introduction}

Many patients with severe organ failure would benefit from organ transplantation, which would result in an improved quality of life and prolong their survival. However, the availability of suitable allografts limits access to organ transplantation and results in substantial waiting lists with a significant mortality during the waiting period. Recently-introduced technologies, especially interspecies chimaeras generated with pluripotent stem cells (PSC) or induced PSC (iPSC), suggest that it will soon be possible to produce human organs in genetically-modified chimaeric pigs [1-3]. iPSC derived from the patient who needs an organ could be injected into a genetically-modified pig embryo, enabling a human organ to develop which can subsequently be transplanted into the patient [4,5]. However, researchers face numerous difficulties in making human organs with this approach because the evolutionary distance between pig and human could prevent the successful development of interspecies blastocyst chimaeras. Other potential problems include the ethical issues of human-pig chimaeras and the potential for the generation of hybrid human-pig viruses. With the recent development of CRISPR/Cas9 technology, which significantly increases gene-editing efficiency, it is possible to test the approach of producing human organs through a combination of CRISPR/Cas9 and iPSC technologies.

\section{Designer Endonucleases as a Tool for Precise and Efficient Gene Editing}

A series of studies demonstrated that designer endonucleases, such as zinc finger nucleases (ZFNs) and transcription activator-like effector nucleases (TALENs), enable genetic modifications to be made by inducing DNA double-strand breaks that stimulate error-prone nonhomologous end joining or homology-directed repair at specific genomic locations [6] (Figures 1 and 2A,B). ZFNs and TALENs recognize specific DNA sequences by protein-DNA interactions and induce DNA site-specific lesions through the dimeric nuclease domain of FokI. Each of these platforms, however, has unique limitations (Table 1). Although multiple strategies have been developed to overcome many limitations, fusing of functional ZFNs and TALENs still requires a time-consuming screening process [7-14]. 


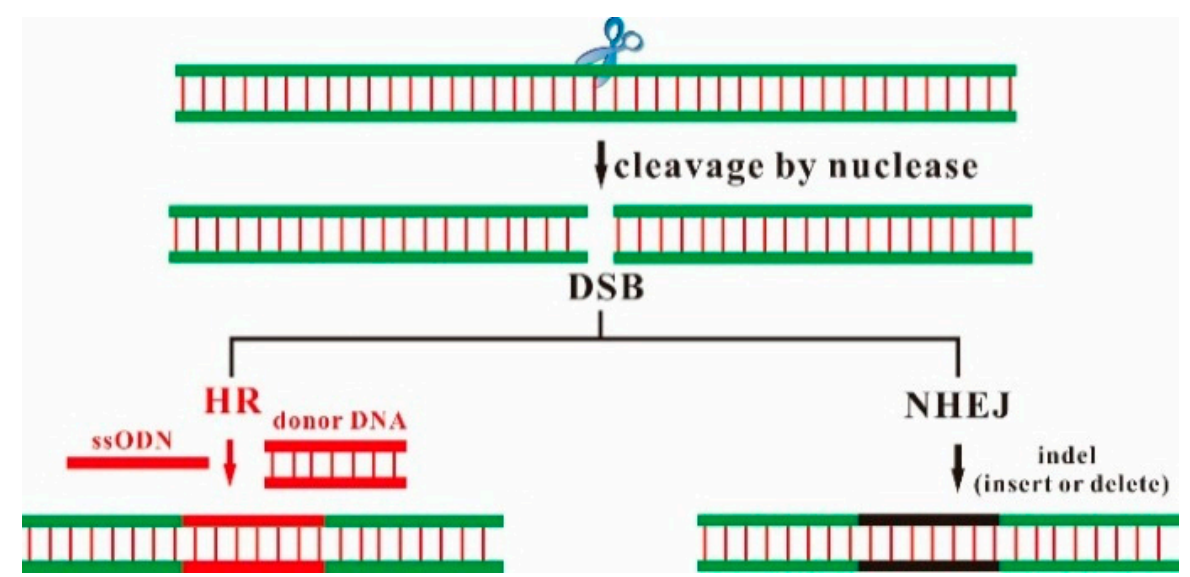

(A)

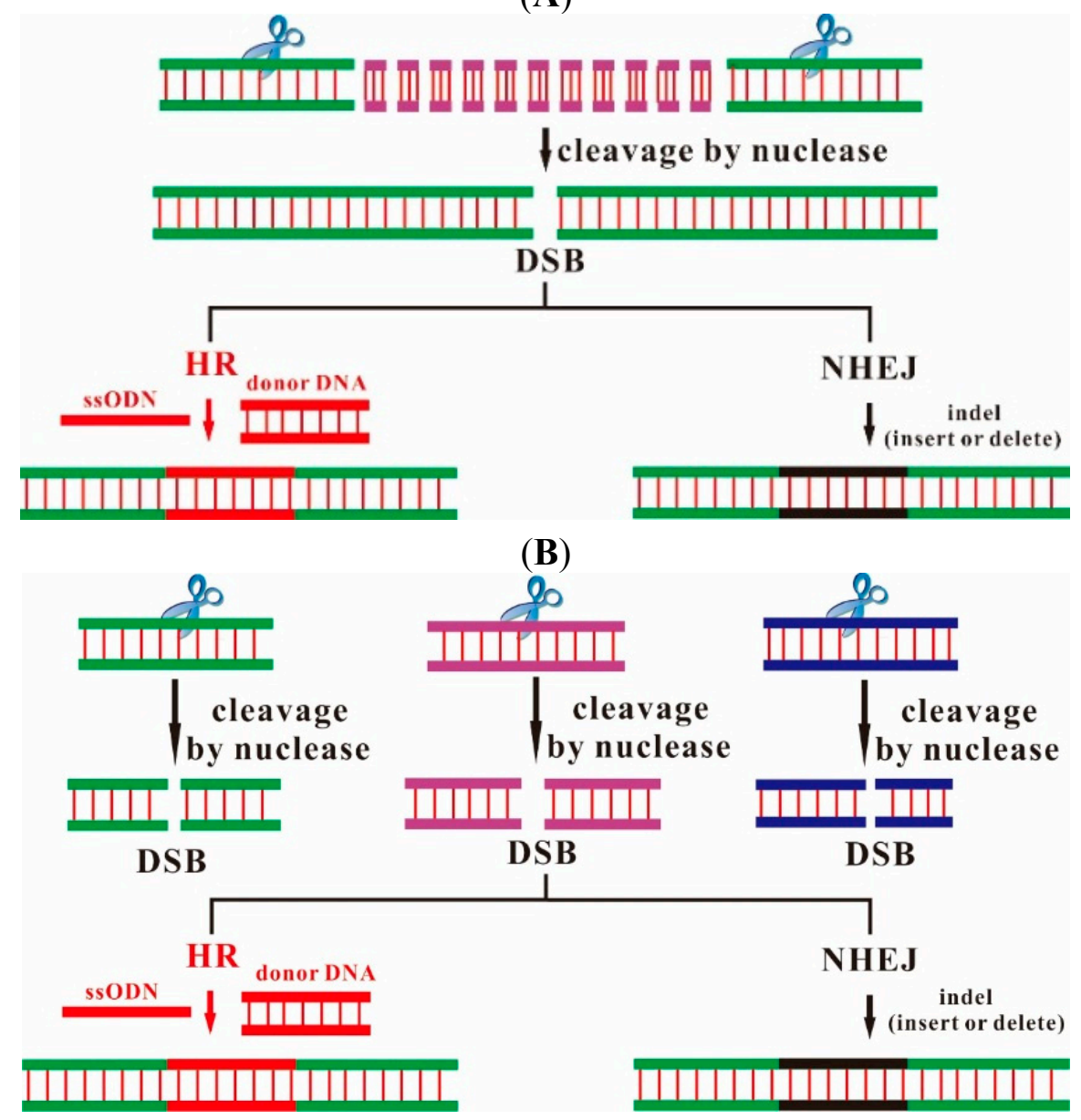

(C)

Figure 1. Outcome of genome editing used nucleases. Nuclease-induced DNA double-strand breaks (DSBs) can lead to sequence indels (insertion or deletion; black) through non-homologous end-joining (NHEJ) or nucleotide correction (red) through homology-directed repair (HR) in the presence of a donor DNA or a single-strand oligodeoxynucleotide (ssODN). (A) single gene editing; (B) long sequence deletion; and (C) multiple gene editing. 

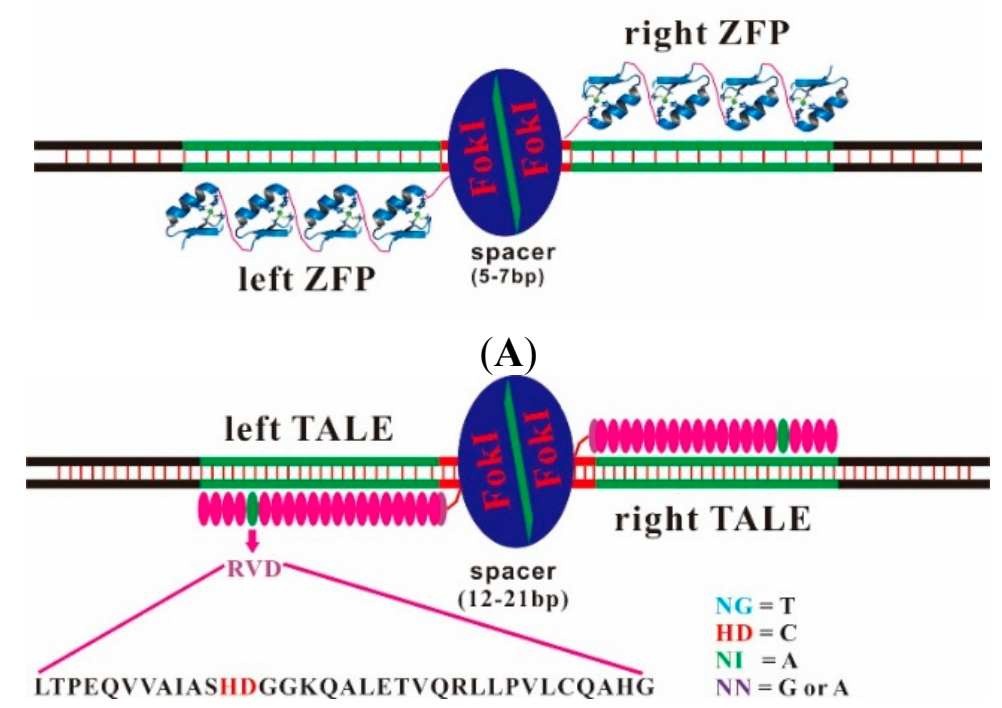

(B)

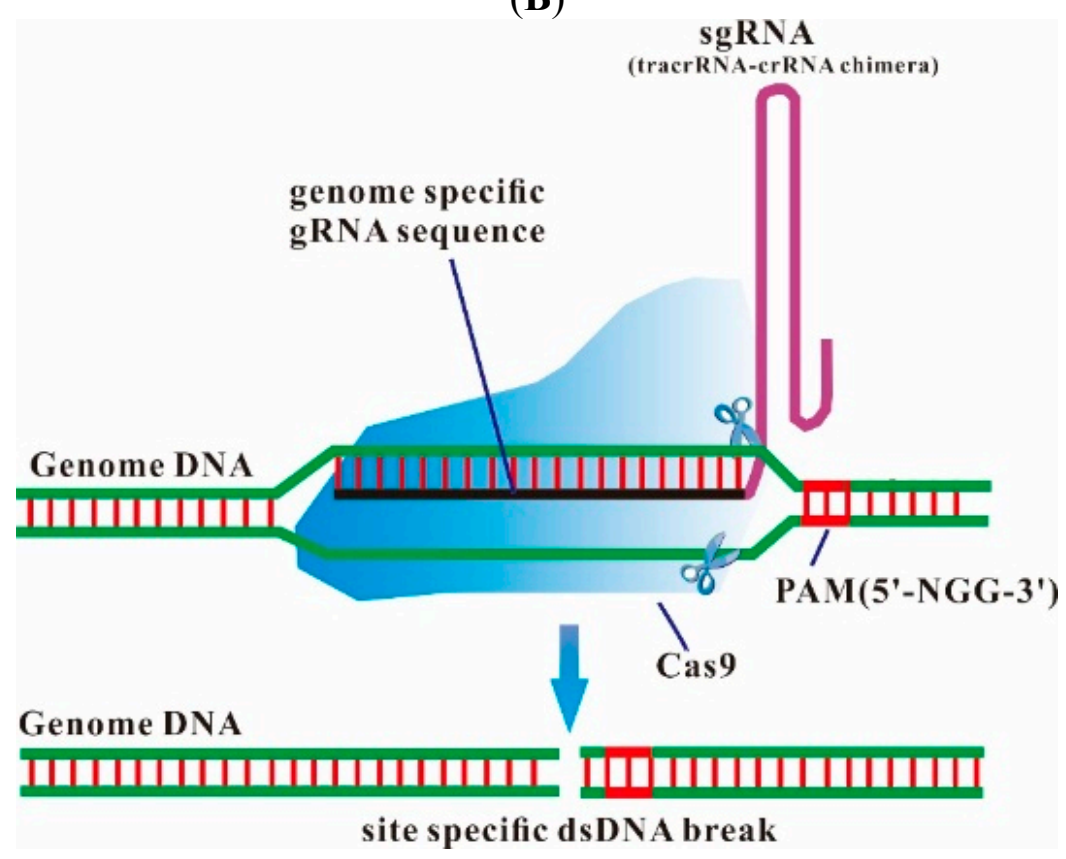

(C)

Figure 2. Schematic representation of (A) ZFN, (B) TALEN, and (C) CRISPR/Cas9. (A) Each ZFN is composed of different zinc-finger proteins (ZFP) at the amino terminus and of the FokI nuclease domain at the carboxyl terminus. Each ZFP recognizes three base pairs; (B) Each transcription activator-like effector nuclease (TALEN) is composed of a transcription activator-like effector (TALE) at the amino terminus and the FokI nuclease domain at the carboxyl terminus. Each TALE repeat is comprised of 33-35 amino acids and recognizes a single base pair through the amino acids at positions 12 and 13, which is called the repeat variable diresidue (RVD, shown in red); and (C) CRISPR/Cas9 is composed of Cas9 protein and a single-chain guide RNA (sgRNA). The guide sequence in the crRNA (shown in black) is complementary to a 20-bp target DNA sequence known as a protospacer, which is next to the 5'-NGG-3'. 
The recent development of the CRISPR/Cas9 genome editing system, employing components of the bacterial adaptive immune response pathway, does not require custom protein synthesis, and instead uses a unique guide RNA along with a single endonuclease protein (Cas9) [15] (Table 1 and Figure 2C).

Table 1. Comparison of intrinsic technical performance for ZFN, TALEN and CRISPR/Cas9.

\begin{tabular}{cccc}
\hline Factors & ZFN & TALEN & CRISPR/Cas9 \\
\hline Nuclease construction & significant & significant & simple \\
In vitro testing & significant & significant & simple \\
Target-efficient & limiting factor & average & good \\
Off-target-efficient & high & low & low \\
Target site choose & limited & limited & unlimited \\
Multiple gene mutations & limited & limited & unlimited \\
Designed component & protein & protein & RNA \\
Essential components & zinc finger proteins & TALE and FokI & guid RNA + Cas9 protein \\
Time consumption & FokI fusion protein & fusion protein & short (1-3 days) \\
Cost & long (7-15 days) & long (5-7 days) & low \\
\hline
\end{tabular}

\section{From Bacterial CRISPR Immune Systems to Engineered RNA-Guided Endonucleases (CRISPR/Cas9)}

The CRISPR story began in 1987, when Ishino and coworkers discovered an unusual structure of repetitive DNA downstream from the Escherichia coli iap gene consisting of invariant direct repeats and variable spacing sequences; these invariant direct repeats were interspaced by five intervening variable spacing sequences [16]. Because of this feature, they received the name CRISPR (clustered regulatory interspaced short palindromic repeats). Furthermore, these CRISPR cassettes are located in close proximity to the CRISPR associated genes (Cas), the protein products of which have helicase and nuclease activity. Over 20 years, the basic function and mechanisms of CRISPR/Cas systems in bacteria have become clear. It has been proposed that CRISPR/Cas is an adaptive defense system that might use antisense RNAs as memory signatures of previous bacteriophage infection by exploiting Watson-Crick base pairing between nucleic acids. During the adaptation stage, resistance is acquired by integration of a new spacer sequence in a CRISPR array. During the expression stage, CRISPR arrays are then transcribed and processed into small RNAs (crRNAs) and Cas proteins. In the late interference stage, the crRNA guide Cas9 proteins to cleave complementary nucleic acids [17,18].

A key advance was the dual tracrRNA:crRNA in 2012, which was engineered as a single-guide RNA (suit guide RNA, sgRNA) that retained initial functionality. The 20-nucleotide sequence at the 5' end of the sgRNA determines the DNA target site by Watson-Crick base pairing, and the double-stranded structure at the 3' side of the guide sequence binds Cas9 to cleave any DNA sequence of interest, as long as it is adjacent to a protospacer-adjacent motif (PAM) (Figure 2C) [19]. In contrast to ZFNs and TALENs, which require substantial protein engineering and a time-consuming screening process for each DNA sequence of interest, the CRISPR/Cas9 system requires only a change in a 20-nucleotide sequence at the 5' end of the sgRNA [20-22]. The CRISPR/Cas9 technology has been rapidly and widely adopted to target genome editing of a vast array of cells and animals [23,24]. 


\section{Rapid and Efficient Generation of Genetically-Modified Animals}

CRISPR/Cas9-mediated genome editing has enabled accelerated generation of genetically-modified animals. In 2012, Jinek et al. demonstrated that dual tracrRNA:crRNA directed the CRISPR/Cas9 to introduce double-stranded breaks in the target DNA in vitro [19]. Three studies in January 2013 showed that CRISPR/Cas9 represented an efficient tool to edit the genomes of human cells with humanized versions of Streptococcus pyogenes Cas9 [20-22]. CRISPR/Cas9-mediated editing has been applied to various cells and animals [25-33]. For rapid and efficient generation of genetically-modified animals, Cas9 can be easily introduced into the target cells using transient transfection of plasmids which carry Cas9 and the appropriately designed sgRNA, followed by somatic cell nuclear transfer (SCNT) [32].

An alternative method involves Cas9 and sgRNA transcribed into mRNA in vitro and directly injected into fertilized zygotes to achieve heritable gene modification at one or multiple alleles (Figure1A,C) [30,34,35]. In order to simply and quickly develop genome editing in mouse models, four recent studies described a more convenient method of model generation using the CRISPR/Cas9 system in vivo in wild-type mice [36-39]. In 2014, Hai and Whitworth and their respective colleagues showed that zygote injection of the Cas9 and sgRNA mRNA efficiently generated genome-modified pigs in one step [29,34]. Rapid and efficient CRISPR/Cas9-mediated genome editing in pigs has opened up unlimited possibilities of genetic engineering in large animals for applications in regenerative medicine.

\section{Making Human Organs from Human iPSCs and Genetically-Engineered Chimaeric Pigs}

Four main methods of providing functional organs for humans have been reported in recent years: (i) creation of organs in vitro in the laboratory ("lab-dish" organs) from PSC or iPSC [40-44]; (ii) the construction of bionic organs in vitro [45-47]; (iii) ex vivo decellularization and recellularization of human or pig organs through regenerative techniques [48-52]; and (iv) genetic engineering of pigs to render their organs resistant to the human immune response (xenotransplantation) [53-55]. The in vitro generation of organs derived from PSC and iPSC or the construction of bionic organs is complex. Blastocyst complementation, first reported by Chen et al., can provide the organ with a "developmental niche" in vivo, generating an almost entirely iPSC-derived organ [56]. They showed that deficiency of T and B lymphocyte lineages in Rag2-deficient mice was complemented by injecting normal mouse embryonic stem cells into mutant Rag2 mouse-derived blastocysts.

To examine the potential for xenogeneic approaches in blastocyst complementation, the groups of Nakauchi and Okabe demonstrated that interspecies chimaeras can be used for the generation of an entire organ from PSCs or iPSC [57,58]. In these studies, the researchers injected rat iPSC into a mutant mouse, which would normally be born lacking a pancreas or thymus, but the injection of wild-type rat iPSCs allowed the development of rat organs in these chimaeras. In theory, interspecies hybrids can be used to generate any tissue or organ type, regardless of its complexity. In 2013, Nakauchi and colleagues successfully generated a pig pancreas by blastocyst complementation, indicating that this approach is successful in a large animal [1].

The feasibility of blastocyst complementation using cloned porcine embryos allows experimentation toward the in vivo generation of functional organs from xenogeneic PSCs in large animals. Following Nakauchi's study, Kim reported that Rag2-mutant pigs (produced through TALENs, with a T-B-NK ${ }^{+}$ 
SCID phenotype) support proliferation and differentiation of human iPSCs and allogeneic porcine trophoblast stem cells [3]. However, it has yet to be proved that human organs can be generated in pigs by blastocyst complementation. In addition, to completely overcome the species barrier, with the associated risks of rejection and/or cross-species infection, one problem that is still to be overcome is the need to humanize the animal's vascular system, which would still express pig antigens (Figure 3). This goal could be achieved by producing multi-gene mutations of essential regulators of vascular and lymphatic tissues in the desired organ via rapid and efficient CRISPR/Cas9-mediated genome editing in concert with blastocyst complementation.

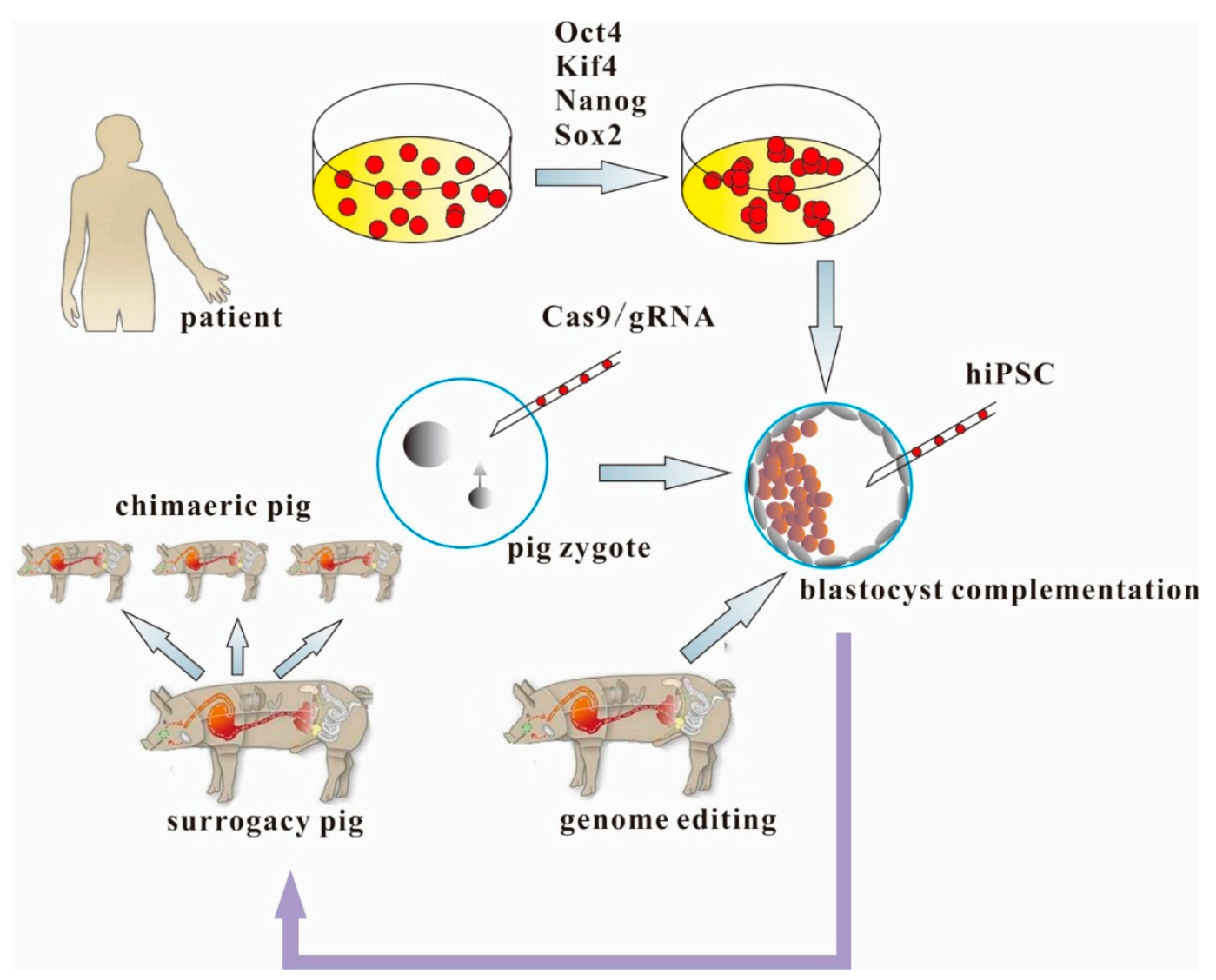

Figure 3. Combination of CRISPR/Cas9 and pluripotent stem cells to provide human organs from chimaeric pigs. Generation of human organs by producing multigene mutations of essential regulators of vascular and lymphatic tissues in the desired organ via rapid and efficient CRISPR/Cas9-mediated genome editing in concert with blastocyst complementation.

However, two questions still remain in this field. First, the currently-available human iPSCs are so-called "primed" and do not develop chimeras when injected into blastocysts. It will be necessary to generate naïve human iPSCs to develop human/pig chimeras [59-61]. Second, safety and ethical issues remain in respect to developing human/pig chimeras. Human iPSC-derived chimeras would possibly carry human neural and germ cells, which evokes ethical controversy. However, with the advantage of CRISPR/Cas9, forced expression of specific genes can be used to guide human iPSC to target organs after blastocyst injection. This method has been successfully used to generate functional pancreas in pancreatogenesis-disabled Pdx1-knockout mice $[5,62]$. 


\section{Conclusions}

Organs from humanized chimaeric pigs that have undergone CRISPR/Cas9 system-mediated gene editing in concert with multigene blastocyst complementation have the potential to resolve the current problem of organ availability for purposes of transplantation. Furthermore, this combined technology could supply organs that will not trigger any immune response, and potentially save hundreds of thousands of lives each year. However, numerous challenges remain. Close collaboration between scientists and clinicians, and between academia and industry, will be required if the development of this technology is to succeed.

\section{Acknowledgments}

This work was supported by grants from the Three Outstanding Projects of Shenzhen.

\section{Abbreviations}

CRISPR $=$ Clustered regularly interspaced short palindromic repeats; Cas $=$ CRISPR-associated; PSC $=$ pluripotent stem cells; TALENS = transcription activator-like effector nucleases; ZFNs = zinc-finger nucleases.

\section{Conflicts of Interest}

The authors declare no conflict of interest.

\section{References}

1. Matsunari, H.; Nagashima, H.; Watanabe, M.; Umeyama, K.; Nakano, K.; Nagaya, M.; Kobayashi, T.; Yamaguchi, T.; Sumazaki, R.; Herzenberg, L.A. Blastocyst complementation generates exogenic pancreas in vivo in apancreatic cloned pigs. Proc. Natl. Acad. Sci. USA 2013, 110, 4557-4562.

2. Kang, L.; Wang, J.; Zhang, Y.; Kou, Z.; Gao, S. iPS cells can support full-term development of tetraploid blastocyst-complemented embryos. Cell Stem Cell 2009, 5, 135-138.

3. Lee, K.; Kwon, D.-N.; Ezashi, T.; Choi, Y.-J.; Park, C.; Ericsson, A.C.; Brown, A.N.; Samuel, M.S.; Park, K.-W.; Walters, E.M. Engraftment of human iPS cells and allogeneic porcine cells into pigs with inactivated RAG2 and accompanying severe combined immunodeficiency. Proc. Natl. Acad. Sci. USA 2014, 111, 7260-7265.

4. Shaw, D.; Dondorp, W.; Geijsen, N.; de Wert, G. Creating human organs in chimaera pigs: An ethical source of immunocompatible organs? J. Med. Ethics 2014, doi:10.1136/medethics-2014-102224.

5. Rashid, T.; Kobayashi, T.; Nakauchi, H. Revisiting the flight of icarus: Making human organs from PSCs with large animal chimeras. Cell Stem Cell 2014, 15, 406-409.

6. Gaj, T.; Gersbach, C.A.; Barbas, C.F., III. ZFN, TALEN, and CRISPR/Cas-based methods for genome engineering. Trends Biotechnol. 2013, 31, 397-405.

7. Sanjana, N.E.; Cong, L.; Zhou, Y.; Cunniff, M.M.; Feng, G.; Zhang, F. A transcription activator-like effector toolbox for genome engineering. Nat. Protoc. 2012, 7, 171-192. 
8. Joung, J.K.; Sander, J.D. Talens: A widely applicable technology for targeted genome editing. Nat. Rev. Mol. Cell Biol. 2012, 14, 49-55.

9. Zhang, F.; Cong, L.; Lodato, S.; Kosuri, S.; Church, G.M.; Arlotta, P. Efficient construction of sequence-specific TAL effectors for modulating mammalian transcription. Nat. Biotechnol. 2011, 29, 149-153.

10. Reyon, D.; Tsai, S.Q.; Khayter, C.; Foden, J.A.; Sander, J.D.; Joung, J.K. Flash assembly of TALENs for high-throughput genome editing. Nat. Biotechnol. 2012, 30, 460-465.

11. Cermak, T.; Doyle, E.L.; Christian, M.; Wang, L.; Zhang, Y.; Schmidt, C.; Baller, J.A.; Somia, N.V.; Bogdanove, A.J.; Voytas, D.F. Efficient design and assembly of custom TALEN and other TAL effector-based constructs for DNA targeting. Nucleic Acids Res. 2011, 39, e82.

12. Maeder, M.L.; Thibodeau-Beganny, S.; Sander, J.D.; Voytas, D.F.; Joung, J.K. Oligomerized pool engineering (open): An "open-source" protocol for making customized zinc-finger arrays. Nat. Protoc. 2009, 4, 1471-1501.

13. Sander, J.D.; Dahlborg, E.J.; Goodwin, M.J.; Cade, L.; Zhang, F.; Cifuentes, D.; Curtin, S.J.; Blackburn, J.S.; Thibodeau-Beganny, S.; Qi, Y. Selection-free zinc-finger-nuclease engineering by context-dependent assembly (CoDA). Nat. Methods 2011, 8, 67-69.

14. Urnov, F.D.; Miller, J.C.; Lee, Y.-L.; Beausejour, C.M.; Rock, J.M.; Augustus, S.; Jamieson, A.C.; Porteus, M.H.; Gregory, P.D.; Holmes, M.C. Highly efficient endogenous human gene correction using designed zinc-finger nucleases. Nature 2005, 435, 646-651.

15. Mali, P.; Esvelt, K.M.; Church, G.M. Cas9 as a versatile tool for engineering biology. Nat. Methods 2013, 10, 957-963.

16. Ishino, Y.; Shinagawa, H.; Makino, K.; Amemura, M.; Nakata, A. Nucleotide sequence of the iap gene, responsible for alkaline phosphatase isozyme conversion in Escherichia coli, and identification of the gene product. J. Bacteriol. 1987, 169, 5429-5433.

17. Westra, E.R.; Swarts, D.C.; Staals, R.H.; Jore, M.M.; Brouns, S.J.; van der Oost, J. The crisprs, they are a-changin': How prokaryotes generate adaptive immunity. Annu. Rev. Genet. 2012, 46, 311-339.

18. Wiedenheft, B.; Sternberg, S.H.; Doudna, J.A. RNA-guided genetic silencing systems in bacteria and archaea. Nature 2012, 482, 331-338.

19. Jinek, M.; Chylinski, K.; Fonfara, I.; Hauer, M.; Doudna, J.A.; Charpentier, E. A programmable dual-RNA-guided DNA endonuclease in adaptive bacterial immunity. Science 2012, 337, 816-821.

20. Cong, L.; Ran, F.A.; Cox, D.; Lin, S.; Barretto, R.; Habib, N.; Hsu, P.D.; Wu, X.; Jiang, W.; Marraffini, L.A. Multiplex genome engineering using CRISPR/Cas systems. Science 2013, 339, 819-823.

21. Mali, P.; Yang, L.; Esvelt, K.M.; Aach, J.; Guell, M.; DiCarlo, J.E.; Norville, J.E.; Church, G.M. RNA-guided human genome engineering via Cas9. Science 2013, 339, 823-826.

22. Jinek, M.; East, A.; Cheng, A.; Lin, S.; Ma, E.; Doudna, J. RNA-programmed genome editing in human cells. eLife 2013, doi:10.7554/eLife.00471.

23. Hsu, P.D.; Lander, E.S.; Zhang, F. Development and applications of CRISPR-Cas9 for genome engineering. Cell 2014, 157, 1262-1278.

24. Doudna, J.A.; Charpentier, E. The new frontier of genome engineering with CRISPR-Cas9. Science 2014, 346, doi:10.1126/science.1258096. 
25. Friedland, A.E.; Tzur, Y.B.; Esvelt, K.M.; Colaiácovo, M.P.; Church, G.M.; Calarco, J.A. Heritable genome editing in C. elegans via a CRISPR-Cas9 system. Nat. Methods 2013, 10, 741-743.

26. Bassett, A.R.; Tibbit, C.; Ponting, C.P.; Liu, J.-L. Highly efficient targeted mutagenesis of drosophila with the CRISPR/Cas9 system. Cell Rep. 2013, 4, 220-228.

27. Wu, Y.; Liang, D.; Wang, Y.; Bai, M.; Tang, W.; Bao, S.; Yan, Z.; Li, D.; Li, J. Correction of a genetic disease in mouse via use of CRISPR-Cas9. Cell Stem Cell 2013, 13, 659-662.

28. Hruscha, A.; Krawitz, P.; Rechenberg, A.; Heinrich, V.; Hecht, J.; Haass, C.; Schmid, B. Efficient CRISPR/Cas9 genome editing with low off-target effects in zebrafish. Development 2013, 140, 4982-4987.

29. Whitworth, K.M.; Lee, K.; Benne, J.A.; Beaton, B.P.; Spate, L.D.; Murphy, S.L.; Samuel, M.S.; Mao, J.; O'Gorman, C.; Walters, E.M. Use of the CRISPR/Cas9 system to produce genetically engineered pigs from in vitro-derived oocytes and embryos. Biol. Reprod. 2014, doi:10.1095/ biolreprod.114.121723.

30. Li, D.; Qiu, Z.; Shao, Y.; Chen, Y.; Guan, Y.; Liu, M.; Li, Y.; Gao, N.; Wang, L.; Lu, X. Heritable gene targeting in the mouse and rat using a CRISPR-Cas system. Nat. Biotechnol. 2013, 31, 681-683.

31. Yang, D.; Xu, J.; Zhu, T.; Fan, J.; Lai, L.; Zhang, J.; Chen, Y.E. Effective gene targeting in rabbits using RNA-guided Cas9 nucleases. J. Mol. Cell. Biol. 2014, doi:10.1093/jmcb/mjt047.

32. Ni, W.; Qiao, J.; Hu, S.; Zhao, X.; Regouski, M.; Yang, M.; Polejaeva, I.A.; Chen, C. Efficient gene knockout in goats using CRISPR/Cas9 system. PLoS One 2014, 9, e106718.

33. Cho, S.W.; Kim, S.; Kim, J.M.; Kim, J.-S. Targeted genome engineering in human cells with the cas9 RNA-guided endonuclease. Nat. Biotechnol. 2013, 31, 230-232.

34. Hai, T.; Teng, F.; Guo, R.; Li, W.; Zhou, Q. One-step generation of knockout pigs by zygote injection of CRISPR/Cas system. Cell Res. 2014, 24, 372-375.

35. Wang, H.; Yang, H.; Shivalila, C.S.; Dawlaty, M.M.; Cheng, A.W.; Zhang, F.; Jaenisch, R. One-step generation of mice carrying mutations in multiple genes by CRISPR/Cas-mediated genome engineering. Cell 2013, 153, 910-918.

36. Yin, H.; Xue, W.; Chen, S.; Bogorad, R.L.; Benedetti, E.; Grompe, M.; Koteliansky, V.; Sharp, P.A.; Jacks, T; Anderson, D.G. Genome editing with Cas9 in adult mice corrects a disease mutation and phenotype. Nat. Biotechnol. 2014, 32, 551-553.

37. Sánchez-Rivera, F.J.; Papagiannakopoulos, T.; Romero, R.; Tammela, T.; Bauer, M.R.; Bhutkar, A.; Joshi, N.S.; Subbaraj, L.; Bronson, R.T.; Xue, W. Rapid modeling of cooperating genetic events in cancer through somatic genome editing. Nature 2014, 516, 428-431.

38. Maddalo, D.; Manchado, E.; Concepcion, C.P.; Bonetti, C.; Vidigal, J.A.; Han, Y.-C.; Ogrodowski, P.; Crippa, A.; Rekhtman, N.; de Stanchina, E. In vivo engineering of oncogenic chromosomal rearrangements with the CRISPR/Cas9 system. Nature 2014, 516, 423-427.

39. Xue, W.; Chen, S.; Yin, H.; Tammela, T.; Papagiannakopoulos, T.; Joshi, N.S.; Cai, W.; Yang, G.; Bronson, R.; Crowley, D.G. CRISPR-mediated direct mutation of cancer genes in the mouse liver. Nature 2014, 514, 380-384.

40. Antonica, F.; Kasprzyk, D.F.; Opitz, R.; Iacovino, M.; Liao, X.-H.; Dumitrescu, A.M.; Refetoff, S.; Peremans, K.; Manto, M.; Kyba, M. Generation of functional thyroid from embryonic stem cells. Nature 2012, 491, 66-71. 
41. Xia, Y.; Nivet, E.; Sancho-Martinez, I.; Gallegos, T.; Suzuki, K.; Okamura, D.; Wu, M.-Z.; Dubova, I.; Esteban, C.R.; Montserrat, N. Directed differentiation of human pluripotent cells to ureteric bud kidney progenitor-like cells. Nat. Cell Biol. 2013, 15, 1507-1515.

42. Takebe, T.; Sekine, K.; Enomura, M.; Koike, H.; Kimura, M.; Ogaeri, T.; Zhang, R.-R.; Ueno, Y.; Zheng, Y.-W.; Koike, N. Vascularized and functional human liver from an iPSC-derived organ bud transplant. Nature 2013, 499, 481-484.

43. Eiraku, M.; Takata, N.; Ishibashi, H.; Kawada, M.; Sakakura, E.; Okuda, S.; Sekiguchi, K.; Adachi, T.; Sasai, Y. Self-organizing optic-cup morphogenesis in three-dimensional culture. Nature 2011, 472 , $51-56$.

44. Nakano, T.; Ando, S.; Takata, N.; Kawada, M.; Muguruma, K.; Sekiguchi, K.; Saito, K.; Yonemura, S.; Eiraku, M.; Sasai, Y. Self-formation of optic cups and storable stratified neural retina from human ESCs. Cell Stem Cell 2012, 10, 771-785.

45. Mannoor, M.S.; Jiang, Z.; James, T.; Kong, Y.L.; Malatesta, K.A.; Soboyejo, W.O.; Verma, N.; Gracias, D.H.; McAlpine, M.C. 3D printed bionic ears. Nano Lett. 2013, 13, 2634-2639.

46. Murphy, S.V.; Atala, A. 3D bioprinting of tissues and organs. Nat. Biotechnol. 2014, 32, 773-785.

47. Skardal, A.; Atala, A. Biomaterials for integration with 3-d bioprinting. Ann. Biomed. Eng. 2014, in press.

48. Song, J.J.; Ott, H.C. Organ engineering based on decellularized matrix scaffolds. Trends Mol. Med. 2011, 17, 424-432.

49. Zambon, J.P.; Magalhaes, R.S.; Ko, I.; Ross, C.L.; Orlando, G.; Peloso, A.; Atala, A.; Yoo, J.J. Kidney regeneration: Where we are and future perspectives. World J. Nephrol. 2014, 3, 24-30.

50. Moser, P.T.; Ott, H.C. Recellularization of organs: What is the future for solid organ transplantation? Curr. Opin. Organ Transplant. 2014, 19, 603-609.

51. Soto-Gutierrez, A.; Wertheim, J.A.; Ott, H.C.; Gilbert, T.W. Perspectives on whole-organ assembly: Moving toward transplantation on demand. J. Clin. Investig. 2012, 122, 3817-3823.

52. Madariaga, M.L.; Ott, H.C. Bioengineering kidneys for transplantation. Semin. Nephrol. 2014, 34, 384-393.

53. Ekser, B.; Ezzelarab, M.; Hara, H.; van der Windt, D.J.; Wijkstrom, M.; Bottino, R.; Trucco, M.; Cooper, D.K. Clinical xenotransplantation: The next medical revolution? Lancet 2012, 379, 672-683.

54. Cooper, D.K.; Ekser, B.; Burlak, C.; Ezzelarab, M.; Hara, H.; Paris, L.; Tector, A.J.; Phelps, C.; Azimzadeh, A.M.; Ayares, D.; et al. Clinical lung xenotransplantation-What donor genetic modifications may be necessary? Xenotransplantation 2012, 19, 144-158.

55. Cooper, D.K.; Hara, H.; Ezzelarab, M.; Bottino, R.; Trucco, M.; Phelps, C.; Ayares, D.; Dai, Y. The potential of genetically-engineered pigs in providing an alternative source of organs and cells for transplantation. J. Biomed. Res. 2013, 27, 249-253.

56. Chen, J.; Lansford, R.; Stewart, V.; Young, F.; Alt, F.W. Rag-2-deficient blastocyst complementation: An assay of gene function in lymphocyte development. Proc. Natl. Acad. Sci. USA 1993, 90, 4528-4532.

57. Kobayashi, T.; Yamaguchi, T.; Hamanaka, S.; Kato-Itoh, M.; Yamazaki, Y.; Ibata, M.; Sato, H.; Lee, Y.-S.; Usui, J.-I.; Knisely, A. Generation of rat pancreas in mouse by interspecific blastocyst injection of pluripotent stem cells. Cell 2010, 142, 787-799. 
58. Isotani, A.; Hatayama, H.; Kaseda, K.; Ikawa, M.; Okabe, M. Formation of a thymus from rat ES cells in xenogeneic nude mouse $\leftrightarrow$ rat ES chimeras. Genes Cells 2011, 16, 397-405.

59. Takashima, Y.; Guo, G.; Loos, R.; Nichols, J.; Ficz, G.; Krueger, F.; Oxley, D.; Santos, F.; Clarke, J.; Mansfield, W.; et al. Resetting transcription factor control circuitry toward ground-state pluripotency in human. Cell 2014, 158, 1254-1269.

60. Theunissen, T.W.; Powell, B.E.; Wang, H.; Mitalipova, M.; Faddah, D.A.; Reddy, J.; Fan, Z.P.; Maetzel, D.; Ganz, K.; Shi, L.; et al. Systematic identification of culture conditions for induction and maintenance of naive human pluripotency. Cell Stem Cell 2014, 15, 471-487.

61. Gafni, O.; Weinberger, L.; Mansour, A.A.; Manor, Y.S.; Chomsky, E.; Ben-Yosef, D.; Kalma, Y.; Viukov, S.; Maza, I.; Zviran, A.; et al. Derivation of novel human ground state naive pluripotent stem cells. Nature 2013, 504, 282-286.

62. Kobayashi, T.; Kato-Itoh, M.; Nakauchi, H. Targeted organ generation using Mixl1-inducible mouse pluripotent stem cells in blastocyst complementation. Stem Cells Dev. 2015, 24, 182-189.

(C) 2015 by the authors; licensee MDPI, Basel, Switzerland. This article is an open access article distributed under the terms and conditions of the Creative Commons Attribution license (http://creativecommons.org/licenses/by/4.0/). 